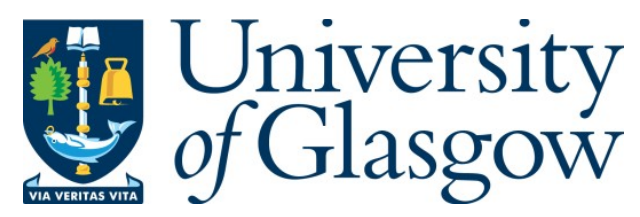

Owens, B. (2020) Smooth, nonsymplectic embeddings of rational balls in the complex projective plane. Quarterly Journal of Mathematics, 71(3), pp. 997-1007.

(doi: 10.1093/qmathj/haaa013)

This is the Author Accepted Manuscript.

There may be differences between this version and the published version. You are advised to consult the publisher's version if you wish to cite from it.

https://eprints.gla.ac.uk/218507/

Deposited on: 19 June 2020

Enlighten - Research publications by members of the University of Glasgow http://eprints.gla.ac.uk 


\title{
SMOOTH, NONSYMPLECTIC EMBEDDINGS OF RATIONAL BALLS IN THE COMPLEX PROJECTIVE PLANE
}

\author{
BRENDAN OWENS
}

\begin{abstract}
We exhibit an infinite family of rational homology balls which embed smoothly but not symplectically in the complex projective plane. We also obtain a new lattice embedding obstruction from Donaldson's diagonalisation theorem, and use this to show that no two of our examples may be embedded disjointly.
\end{abstract}

\section{INTRODUCTION}

A Markov triple is a positive integer solution $\left(p_{1}, p_{2}, p_{3}\right)$ to the Markov equation

$$
p_{1}^{2}+p_{2}^{2}+p_{3}^{2}=3 p_{1} p_{2} p_{3} .
$$

Each Markov triple gives rise to an embedding

$$
\bigsqcup_{i=1}^{3} B_{p_{i}, q_{i}} \hookrightarrow \mathbb{C P}^{2}
$$

of a disjoint union of three rational homology balls in the complex projective plane. Here $B_{p, q}$ is the rational homology ball smoothing of the quotient singularity $\frac{1}{p^{2}}(1, p q-$ 1). The embedding in (2) arises by smoothing the three singular points in the weighted projective space $\mathbb{P}\left(p_{1}^{2}, p_{2}^{2}, p_{3}^{2}\right)$, and the numbers $q_{i}$ are given by

$$
q_{i}= \pm 3 p_{j} / p_{k} \quad\left(\bmod p_{i}\right)
$$

where $i, j, k$ is a permutation of $1,2,3$. The apparent sign ambiguity here is due to the fact that $B_{p, q} \cong B_{p, p-q}$.

Hacking and Prokhorov proved in [5] that any projective surface with quotient singularities which admits a smoothing to $\mathbb{C P}^{2}$ is $\mathbb{Q}$-Gorenstein deformation equivalent to some $\mathbb{P}\left(p_{1}^{2}, p_{2}^{2}, p_{3}^{2}\right)$ as above. Evans and Smith proved in [4] that any disjoint union $\bigsqcup_{i \in \mathcal{I}} B_{p_{i}, q_{i}}$ which admits a symplectic embedding in $\mathbb{C P}^{2}$ arises in this way, with $|\mathcal{I}| \leq 3$.

Let $F(2 n-1)$ denote the $n$th odd Fibonacci number, defined by the recursion

$$
F(2 n+3)=3 F(2 n+1)-F(2 n-1), \quad F(1)=1, \quad F(3)=2 .
$$

Then (1, $F(2 n-1), F(2 n+1))$ is a Markov triple for each $n \in \mathbb{N}$, showing in particular that $B_{F(2 n+1), F(2 n-3)}$ admits a symplectic embedding in $\mathbb{C P}^{2}$ for each $n>1$.

Date: June 18, 2020. 
In [8] we mentioned but overlooked the significance of the following result. Here $\Delta_{p, q}$ is a properly embedded surface in the 4-ball whose double branched cover is $B_{p, q}$, and $P_{+}$is the unknotted Möbius band in the 4-ball with normal Euler number 2; see [8] for further details.

Theorem 1. For each $n \in \mathbb{N}$, the slice surface $\Delta_{F(2 n+1), F(2 n-1)}$ admits a simple embedding as a sublevel surface of the unknotted Möbius band $P_{+}$. Taking double branched covers yields a simple smooth embedding

$$
B_{F(2 n+1), F(2 n-1)} \hookrightarrow \mathbb{C P}^{2} .
$$

If $n>1$, then $B_{F(2 n+1), F(2 n-1)}$ does not embed symplectically in $\mathbb{C P}^{2}$.

Theorem 1 gives the first-known smooth embeddings of rational balls $B_{p, q}$ in the complex projective plane that do not arise from symplectic embeddings. This shows that the smooth embedding problem has an as-yet-unknown solution which differs from that to the symplectic problem solved by Evans-Smith. Bulent Tosun has informed the author that work of Nemirovski-Segal [7] implies the existence of a rational ball, bounded by a Seifert fibred space with 3 exceptional fibres, which embeds smoothly but not sympectically in $\mathbb{C P}^{2}$. Most of the embeddings obtained in [8], but not those given in Theorem 1 , have since been reproved and generalised by different methods in [9].

A conjecture of Kollár [6] would imply that at most three rational balls $B_{p_{i}, q_{i}}$ may embed smoothly and disjointly in $\mathbb{C P}^{2}$. The following result gives some mild support to this conjecture.

Theorem 2. It is not possible to smoothly embed a disjoint union $\bigsqcup_{i \in \mathcal{I}} B_{p_{i}, q_{i}}$ of two or more of the balls from Theorem 1 in $\mathbb{C P}^{2}$, where each $\left(p_{i}, q_{i}\right)$ is a consecutive pair of odd Fibonacci numbers.

This result uses a new obstruction derived from Donaldson's diagonalisation theorem [3]. This is stated in Proposition 3.2.

Corrigendum to [8]. In [8, sentence after Theorem 5, and Remark 4.1] we incorrectly stated that $B_{F(2 n+1), F(2 n-1)}$ embeds symplectically in $\mathbb{C P}^{2}$. I am very grateful to Giancarlo Urzúa who reminded me that the Markov triple $(1, F(2 n-1), F(2 n+1))$ gives rise to a symplectic embedding in $\mathbb{C P}^{2}$ of $B_{F(2 n+1), F(2 n-3)}$, and not of $B_{F(2 n+1), F(2 n-1)}$.

Further acknowledgements. I am grateful to Jonny Evans, Marco Golla, Ana Lecuona, Yankı Lekili, Duncan McCoy, Bulent Tosun, and Giancarlo Urzúa for helpful comments and conversations. I also thank the anonymous referee for helpful suggestions.

\section{Smooth EMBEDdings}

In this section we prove Theorem 1, using the method from [8]. 
We refer the reader to [1] for an excellent and readable source on Markov numbers. Suppose that $(p, a, b)$ is a solution to the Markov equation (1) with $p>a, b$. By $[1$, Corollary 3.4], the integers in a Markov triple are pairwise relatively prime, so that there are unique solutions $x=u, u^{\prime}$ to

$$
b \equiv \pm x a \quad(\bmod p)
$$

These satisfy $u+u^{\prime} \equiv 0(\bmod p)$, so that one of them (say $\left.u\right)$ is between 0 and $p / 2$; we call this number $u$ the characteristic number of the Markov triple $(p, a, b)$. The Markov equation gives $a^{2}+b^{2} \equiv 0(\bmod p)$, from which it follows that

$$
u^{2} \equiv-1 \quad(\bmod p)
$$

I am grateful to Jonny Evans for helping me to see the following result.

Lemma 2.1. Let $n \in \mathbb{N}$. The rational ball $B_{F(2 n+1), F(2 n-1)}$ embeds symplectically in $\mathbb{C P}^{2}$ if and only if $n=1$.

Proof. From [4, Theorem 4.15] we have that $B_{p, q}$ embeds symplectically in $\mathbb{C P}^{2}$ if and only if $p$ is the maximum of a Markov triple $(a, b, p)$, and $q= \pm 3 b / a(\bmod p)$. Then in fact $q= \pm 3 u$, where $u$ is the characteristic number of the Markov triple.

For $n>1$, the odd Fibonacci number $F(2 n+1)$ is the maximum of the Markov triple $(1, F(2 n-1), F(2 n+1))$, from which it follows that $B_{F(2 n+1), F(2 n-3)}$ embeds symplectically. Also note that the characteristic number of this Markov triple is $F(2 n-1)$, and $F(2 n-1)^{2} \equiv-1(\bmod F(2 n+1))$.

Then $B_{F(2 n+1), F(2 n-1)}$ embeds symplectically if and only if $F(2 n+1)$ is the maximum of another Markov triple $(a, b, F(2 n+1))$, and $F(2 n-1)= \pm 3 u$, where $u$ is the characteristic number of the triple $(a, b, F(2 n+1))$. This would imply that

$$
-1 \equiv F(2 n-1)^{2} \equiv 9 u^{2} \equiv-9 \quad(\bmod F(2 n+1)) .
$$

The only odd Fibonacci numbers which divide 8 are $F(1)=1$ and $F(3)=2$, so we conclude that $n=1$.

Finally, $F(3)=2$ is the maximum of the Markov triple $(1,1,2)$ and $B_{F(3), F(1)}=B_{2,1}$ does embed sympectically.

Proof of Theorem 1. As noted in the proof of Lemma 2.1, the Markov triple $(1,1,2)$ gives rise to an embedding of $B_{F(3), F(1)}=B_{2,1}$ in $\mathbb{C P}^{2}$. Suppose now that $n>1$. Induction using (3) yields the Hirzebruch-Jung continued fraction expansion

$$
\frac{F(2 n+1)}{F(2 n-1)}=\left[3^{n-1}, 2\right] .
$$

Now using [8, Lemma 3.1] we have

$$
\frac{F(2 n+1)^{2}}{F(2 n+1) F(2 n-1)-1}=\left[3^{n-1}, 5,3^{n-2}, 2\right] .
$$


These continued fractions may be used to describe the surface $\Delta_{F(2 n+1), F(2 n-1)}$, as described in [8].

The proof that $\Delta_{F(2 n+1), F(2 n-1)}$ is a sublevel surface of $P_{+}$is a minor modification of the proof of [8, Theorem 5]. We refer the reader to that source for details.

Consider the first diagram shown in Figure 1. This represents a surface $\Sigma$ bounded by the unknot, which we claim is $P_{+}$. Note first that the band move corresponding to the blue band labelled 0 converts the diagram to one of $\Delta_{F(2 n+1), F(2 n-1)}$, which is the slice disk described by Casson and Harer [2] for the two-bridge knot $S(F(2 n+$ $\left.1)^{2}, F(2 n+1) F(2 n-1)-1\right)$. This shows that $\Delta_{F(2 n+1), F(2 n-1)}$ is a sublevel surface of the surface $\Sigma$. It remains to see that $\Sigma$ is the unknotted Möbius band $P_{+}$whose double branched cover is $\mathbb{C P}^{2}$ minus a 4 -ball.
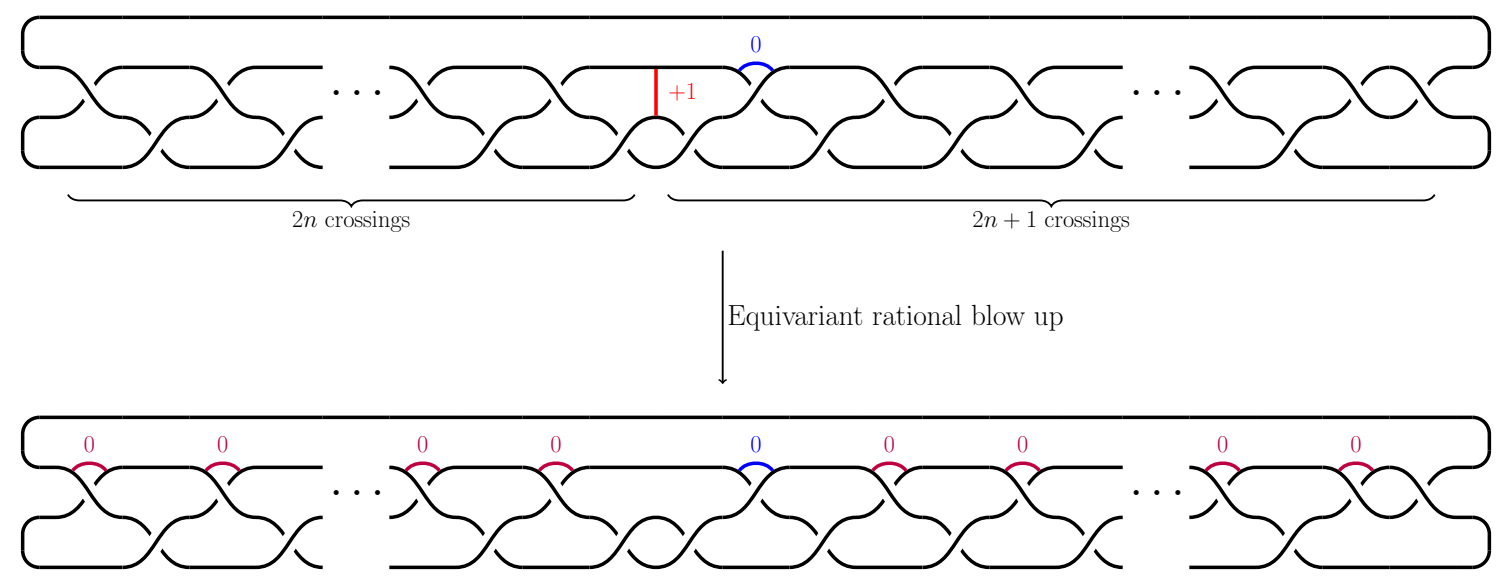

FIGURE 1. The slice disk $\Delta_{F(2 n+1), F(2 n-1)}$ as a sublevel surface of $P_{+}$, and the resulting equivariant rational blow up. Numbers beside bands give the signed count of half-twists or crossings.

Figure 2 shows a sequence of isotopies and band slides converting $\Sigma$ to $P_{+}$in the first case of interest which is $n=2$. Taking double branched covers we see that $B_{5,2}$ admits a smooth embedding in $\mathbb{C P}^{2}$. The proof for $n>2$ follows by an induction argument involving band slides similar to those in Figure 2. The inductive step is shown in Figure 3.

Recall that an embedding of $B_{p, q}$ in a 4-manifold $Z$ is called simple if the resulting rational blow up of $Z$ is obtainable by a sequence of ordinary blow ups. The proof that the embeddings described above are simple follows as in [8, Proposition 5.1]; we again refer the reader to [8] for more details on equivariant rational blow up, and to Section 3 for a description of rational blow up. We describe here a slightly shorter version of the proof at the level of double branched covers. The second diagram in Figure 1 represents the surface in the 4-ball pushed in from the black surface of the 

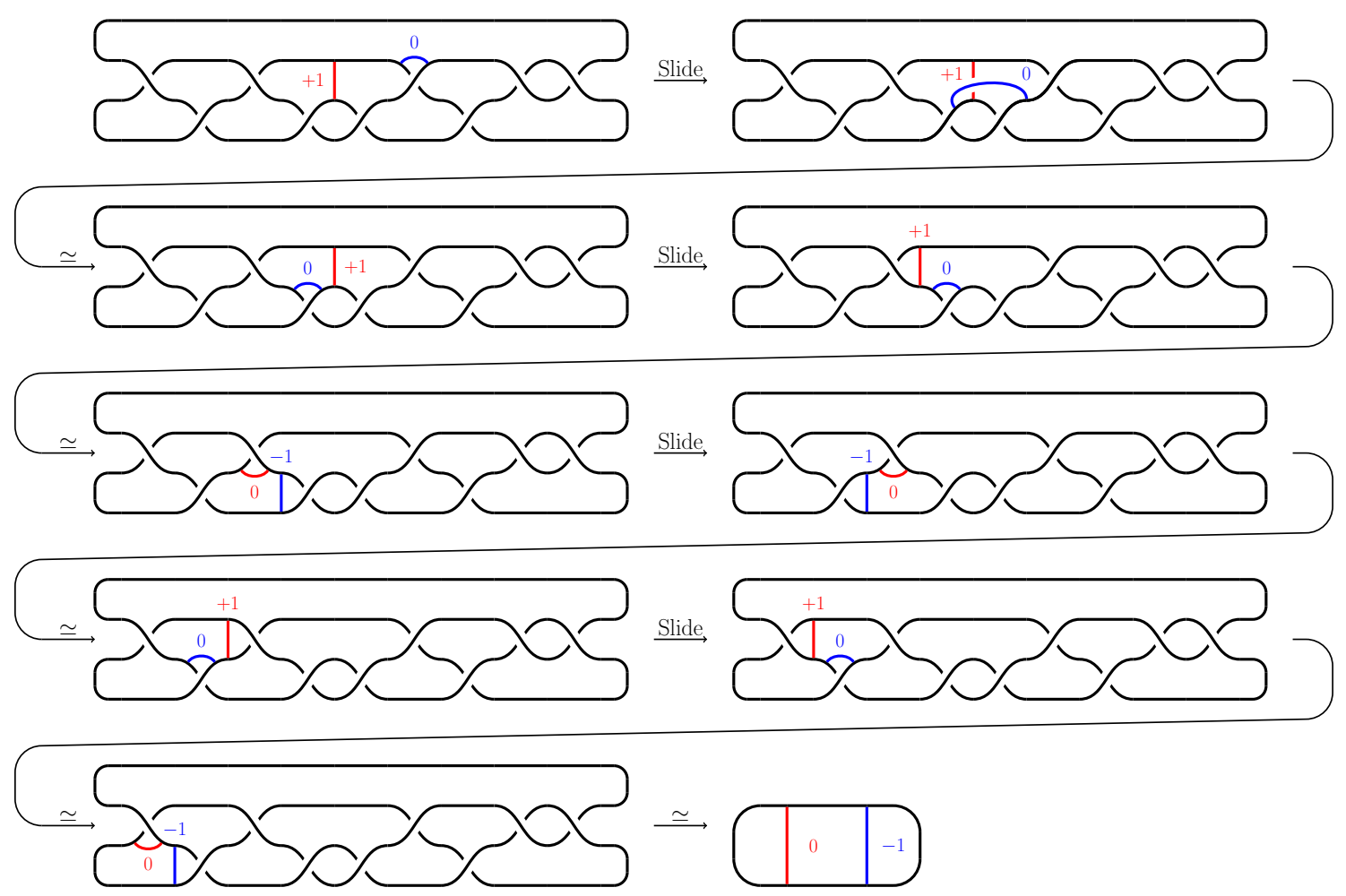

Figure 2. The slice disk $\Delta_{5,2}$ is a sublevel surface of $P_{+}$.

two-bridge diagram shown, using a chessboard colouring in which the unbounded region is white. The rational blow up of $\mathbb{C P}^{2}$, minus a 4-ball, is the double cover $X$ of the 4-ball branched along this black surface, which in turn is the plumbing of disk bundles over $S^{2}$ corresponding to the linear graph with weights

$$
(-3)^{n-1},-2,-1,(-3)^{n-2},-2
$$

where $(-3)^{m}$ denotes -3 repeated $m$ times. A sequence of -1 blow downs reduces this to the linear plumbing with weights -3 and 0 , which is diffeomorphic to $\mathbb{C P}^{2} \# \overline{\mathbb{C P}^{2}}$, again minus a ball. It follows that

$$
X \cong \mathbb{C P}^{2} \#(2 n-1) \overline{\mathbb{C P}^{2}}
$$

Together with Lemma 2.1, this completes the proof of Theorem 1. 


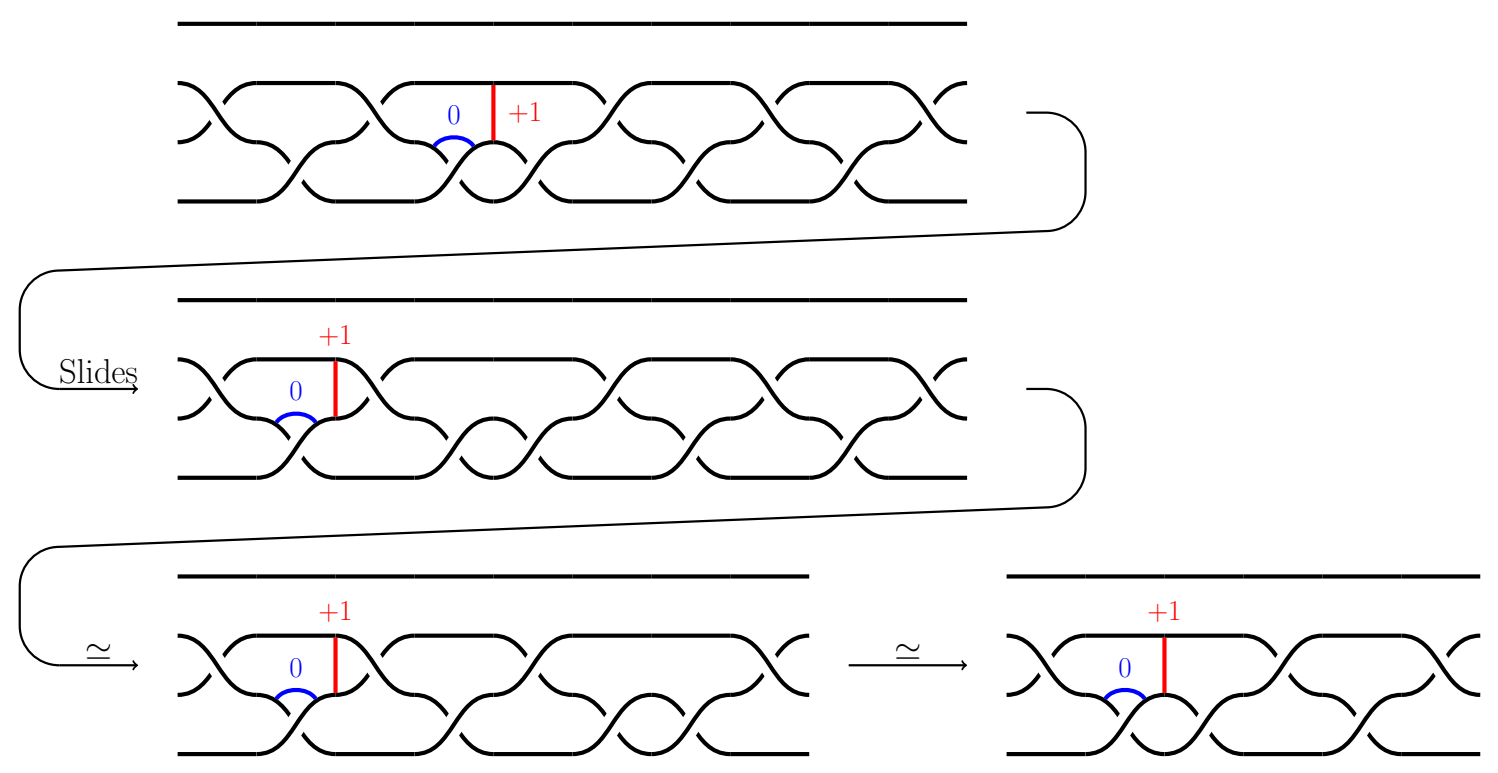

Figure 3. The inductive step. The band slides are similar to those shown in Figure 2. This shows how to transform the first diagram in Figure 1 with $n=k$, to the same diagram with $n=k-1$.

\section{An obstruction from Donaldson's Diagonalisation theOREM}

In this section, we derive a lattice embedding obstruction to smoothly embedding a rational homology ball bounded by a lens space, or a disjoint union of such, in $\mathbb{C P}^{2}$. We begin by setting some conventions and terminology.

All homology and cohomology groups in this section have integer coefficients. Recall that if $X$ is a smooth 4-manifold, possibly with boundary, then its intersection lattice $\Lambda_{X}$ consists of the free abelian group $H_{2}(X) /$ Tors together with the symmetric bilinear intersection pairing. The term lens space will be used here to refer to $L(p, q)$ with $p>q \geq 1$; in particular not $S^{3}$ or $S^{2} \times S^{1}$. Given integers $a_{1}, \ldots, a_{k}$, the linear lattice $\Lambda\left(a_{1}, \ldots, a_{k}\right)$ is defined to be the free abelian group with generators $v_{1}, \ldots, v_{n}$, and with symmetric bilinear pairing given by

$$
v_{i} \cdot v_{j}= \begin{cases}a_{i} & \text { if } i=j \\ -1 & \text { if }|i-j|=1 \\ 0 & \text { if }|i-j|>1\end{cases}
$$

As this is the lattice associated to a weighted linear graph, we often refer to the generators $v_{1}, \ldots, v_{k}$ as vertices. Recall that a lens space $L(p, q)$ is the boundary of a plumbing $C$ of disk bundles over spheres determined by the weighted linear graph 
with weights $a_{1}, \ldots, a_{k} \geq 2$ where

$$
\frac{p}{p-q}=\left[a_{1}, a_{2}, \ldots, a_{k}\right]:=a_{1}-\frac{1}{a_{2}-\ddots_{-\frac{1}{a_{k}}}} .
$$

The intersection lattice of $C$ is then $\Lambda\left(a_{1}, \ldots, a_{k}\right)$.

Let $B$ be a rational homology ball with lens space boundary. Given an embedding $B \hookrightarrow \mathbb{C P}^{2}$, we let $M$ be the complement $\mathbb{C P}^{2} \backslash B$ and "rationally blow up" to obtain the closed positive-definite manifold $M \cup C$, where $C$ is the positive-definite plumbed manifold bounded by $\partial B$. Donaldson's diagonalisation theorem then implies the existence of a lattice embedding

$$
\Lambda_{M} \oplus \Lambda_{C} \hookrightarrow \mathbb{Z}^{m}
$$

where $\Lambda_{M}$ and $\Lambda_{C}$ are the intersection lattices of $M$ and $C$ respectively, and $m$ is the sum of their ranks.

The reader familiar with the use of such lattice obstructions will note that since $M$ is a submanifold of $\mathbb{C P}^{2}$, and since $Y=\partial B$ bounds a rational ball, each of $\Lambda_{M}$ and $\Lambda_{C}$ admit finite-index embeddings in diagonal unimodular lattices, so that an embedding as in (5) must in fact exist, with the first factor embedding in $\mathbb{Z}$ and the second in the orthogonal $\mathbb{Z}^{m-1}$. We will show that simple topological considerations place further restrictions on the lattice embedding in (5), giving rise to a useful obstruction, which also extends to the case of an embedding of a disjoint union of rational balls.

Lemma 3.1. Let $B_{i}$ be rational homology balls bounded by lens spaces for $i=1, \ldots, n$, and suppose that the disjoint union $\bigsqcup_{i} B_{i}$ embeds smoothly in $\mathbb{C P}^{2}$. Then the complement $M=\mathbb{C P}^{2} \backslash \bigsqcup_{i} B_{i}$ has $H_{1}(M ; \mathbb{Z})=0$ and $H_{2}(M ; \mathbb{Z}) \cong \mathbb{Z}$.

Proof. We use the Mayer-Vietoris sequence and induction. The base case is $n=0$ and $M=\mathbb{C P}^{2}$.

Now suppose $M^{\prime}=\mathbb{C P}^{2} \backslash \bigsqcup_{i=1}^{n-1} B_{i}$ has $H_{1}\left(M^{\prime} ; \mathbb{Z}\right)=0$ and $H_{2}\left(M^{\prime} ; \mathbb{Z}\right) \cong \mathbb{Z}$. Then

$$
M^{\prime}=M \cup_{Y} B_{n}
$$

where $Y=L\left(p_{n}^{2}, q_{n}\right)$ has $H_{1}(Y ; \mathbb{Z}) \cong \mathbb{Z} / p_{n}^{2} \mathbb{Z}$. We have $H_{2}\left(B_{n} ; \mathbb{Z}\right)=0$, since it is a torsion subgroup of $H_{2}\left(M^{\prime} ; \mathbb{Z}\right) \cong \mathbb{Z}$; then from the long exact sequence of the pair $\left(B_{n}, Y\right)$, we have $H_{1}\left(B_{n} ; \mathbb{Z}\right) \cong \mathbb{Z} / p_{n} \mathbb{Z}$. The Mayer-Vietoris sequence, with integer coefficients, shows that $H_{2}(M)$ is a finite-index subgroup of $\mathbb{Z}$, hence $H_{2}(M) \cong \mathbb{Z}$. The same sequence shows that there is a surjection from $\mathbb{Z} / p_{n}^{2} \mathbb{Z}$ to $H_{1}(M) \oplus \mathbb{Z} / p_{n} \mathbb{Z}$, from which it follows that the latter direct sum is finite cyclic and also that cyclic summands of $H_{1}(M)$ have orders dividing $p_{n}$. We conclude that $H_{1}(M)$ must be trivial.

We recall the notion of rational blow up, and modify and generalise it for our convenience. If a disjoint union $\bigsqcup_{i} B_{i}$ embeds smoothly in some 4-manifold $Z$, where each $B_{i}$ is a rational ball bounded by a lens space $L\left(p_{i}, q_{i}\right)$, then we may excise each $B_{i}$ 
and replace it by the positive-definite plumbed manifold $C_{i}$ bounded by $L\left(p_{i}, p_{i}-q_{i}\right)$ to obtain a new manifold

$$
X=M \cup C,
$$

called the positive rational blow up of $Z$. Here $M$ is the complement of $\bigsqcup_{i=1}^{n} B_{i}$ in $Z$, and $C$ is the disjoint union $\bigsqcup_{i=1}^{n} C_{i}$ of plumbed manifolds. We assume that all weights in each plumbing $C_{i}$ are at least 2 .

Proposition 3.2. Let $B_{i}$ be rational homology balls bounded by lens spaces for $i=$ $1, \ldots, n$, and suppose that the disjoint union $\bigsqcup_{i} B_{i}$ embeds smoothly in $\mathbb{C P}^{2}$. Let $X=M \cup C$ be the resulting positive rational blow up of $\mathbb{C P}^{2}$. Then there exists a finite-index lattice embedding

$$
\Lambda_{M} \oplus \Lambda_{C} \hookrightarrow \mathbb{Z}^{m}
$$

such that each unit vector $e \in \mathbb{Z}^{m}$ has nonzero pairing with each of $\Lambda_{M}$ and $\Lambda_{C}$. Moreover the image of the generator of $\Lambda_{M}$ is a primitive vector in $\mathbb{Z}^{m}$.

Remark 3.3. Let $A$ be the matrix of the embedding in (6) in terms of a basis $v_{1}, \ldots, v_{m}$ for $\Lambda_{X}$, where $v_{1} \in \Lambda_{M}$ and $v_{2}, \ldots, v_{m} \in \Lambda_{C}$, and an orthonormal basis for $\mathbb{Z}^{m}$. Then the proposition states that each row of $A$ has at least two nonzero entries including one in the first column, and also that the entries of the first column of $A$, which are all nonzero, have no common divisor.

The known embeddings mentioned earlier in this section each give rise to a block diagonal matrix A which does not satisfy the condition in the proposition.

Proof of Proposition 3.2. Let $Y$ denote the union of lens spaces which is the common boundary of $M$ and $C$. Let $e$ be a unit vector in $\Lambda_{X}$. We may write

$$
e=e_{M}+e_{C}
$$

where $e_{M} \in H_{2}(M, Y)$ and $e_{C} \in H_{2}(C, Y)$. There are no unit vectors in $\Lambda_{M}$, which is a rank one lattice whose generator squared is the order of the first homology of $Y$. There are also no unit vectors in $\Lambda_{C}$ since we assumed all weights in each plumbing are at least 2 . It follows that $e_{M}$ and $e_{C}$ are both nonzero.

Since $H_{1}(M)=0$ by Lemma 3.1, all homology groups of $M$ are in fact torsionfree by standard arguments using universal coefficients, Poincaré-Lefschetz duality, and the long exact sequence of the pair. It follows that the second homology group $H_{2}(M)$ is the underlying group of the lattice $\Lambda_{M}$, and the relative homology group $H_{2}(M, Y)$ is the underlying group of the dual lattice $\Lambda_{M}{ }^{*}$ via the universal coefficient theorem. Then since $\Lambda_{M}$ is positive definite, we see that an element of $H_{2}(M, Y)$ is nonzero if and only if it has nonzero intersection with some element in $H_{2}(M)$. Thus in particular $e_{M}$ and also $e$ has nonzero intersection with some element of $H_{2}(M)$. The same argument applies to $\Lambda_{C}$, so that $e_{C}$ and also $e$ has nonzero pairing with some element of $H_{2}(C)$ which is the underlying group of $\Lambda_{C}$. 
Finally let $v$ denote the image in $H_{2}(X)$ of the generator of $\Lambda_{M}$, and suppose that $v=k w$ for some $k \in \mathbb{N}$ and $w \in H_{2}(X)$. As above we write $w=w_{M}+w_{C}$ and we conclude that $w_{C}=0$ since it has zero pairing with all of $\Lambda_{C}$. This implies $w=w_{M} \in \Lambda_{M}$, but then $k=1$ since $v$ is the generator.

In what follows we study lattice embeddings $\Lambda \hookrightarrow \mathbb{Z}^{m}$ up to lattice automorphisms of $\mathbb{Z}^{m}$, or in other words, up to reordering of the orthonormal basis $e_{1}, \ldots, e_{m}$, and/or changing signs of some orthonormal basis elements. Embeddings of linear lattices all of whose weights are 2 or 3 are very restricted, since up to $\operatorname{Aut}\left(\mathbb{Z}^{m}\right)$, vectors $v \in \mathbb{Z}^{m}$ with $v \cdot v=2$ or $v \cdot v=3$ take the form $v=e_{1}+e_{2}$ or $v=e_{1}+e_{2}+e_{3}$.

Example 3.4. The rational ball $B_{3,1}$ does not embed smoothly in $\mathbb{C P}^{2}$.

Proof. The boundary of $B_{3,1}$ is the lens space $L(9,2)$, which also bounds the positivedefinite plumbing $C$ with weights $[2,2,2,3]$. Let $v_{2}, \ldots, v_{5}$ be the generators of the linear lattice $\Lambda_{C}=\Lambda(2,2,2,3)$ as in (4), and let $v_{1}$ be the generator of the rank one lattice $\Lambda_{M}=\Lambda(9)$. Let $e_{1}, \ldots, e_{5}$ be an orthonormal basis for $\mathbb{Z}^{5}$. There is, up to lattice automorphisms of $\mathbb{Z}^{5}$, a unique embedding

$$
\Lambda_{M} \oplus \Lambda_{C} \hookrightarrow \mathbb{Z}^{5}
$$

this takes $v_{1}$ to $3 e_{1}, v_{i}$ to $-e_{i}+e_{i+1}$ for $2 \leq i \leq 4$, and $v_{5}$ to $e_{2}+e_{3}+e_{4}$. This does not satisfy the conditions of Proposition 3.2, since $e_{i}$ has zero pairing with $\Lambda_{M}$ for $i>1$ and $e_{1}$ has zero pairing with $\Lambda_{C}$.

Lemma 3.5. Up to Aut $\left(\mathbb{Z}^{m}\right)$, there are precisely two ways to embed the linear lattice $\Lambda(2,2,2)$ in $\mathbb{Z}^{m}$, where $m \geq 4$. The first has image in a $\mathbb{Z}^{3}$ sublattice of $\mathbb{Z}^{m}$, and its orthogonal complement in this sublattice is the zero sublattice. The second has image in a $\mathbb{Z}^{4}$ sublattice, and its orthogonal complement in $\mathbb{Z}^{4}$ is spanned by a vector $w$ with $w \cdot w=4$.

Let $n>1$ and let $\Lambda$ denote the linear lattice $\Lambda\left(3^{n-1}, 2,2,3^{n-1}, 2\right)$, with rank $r=$ $2 n+1$. Up to Aut $\left(\mathbb{Z}^{m}\right)$, there are precisely three ways to embed $\Lambda$ in $\mathbb{Z}^{m}$, where $m \in \mathbb{N}$ is sufficiently large. The first has image in a $\mathbb{Z}^{r}$ sublattice, and its orthogonal complement in this sublattice is the zero sublattice. The second has image in a $\mathbb{Z}^{r+1}$ sublattice, and its orthogonal complement in $\mathbb{Z}^{r+1}$ is spanned by a vector $w$ with $w \cdot w=$ $F(2 n+1)^{2}$. The third has image in a $\mathbb{Z}^{4 n}$ sublattice, and its orthogonal complement in $\mathbb{Z}^{4 n}$ contains no unit vectors.

Proof. For the first case, we can either map the vertices of $\Lambda(2,2,2)$ to $-e_{1}+e_{2},-e_{2}+$ $e_{3}, e_{1}+e_{2}$ or to $-e_{1}+e_{2},-e_{2}+e_{3},-e_{3}+e_{4}$. It is straightforward to see there are no other possibilities.

In the second case we begin by embedding the two adjacent vertices of weight two. Up to automorphism of $\mathbb{Z}^{m}$, these are mapped to $-e_{1}+e_{2}$ and $-e_{2}+e_{3}$. By inspection, the linear lattice $\Lambda(3,2,2,3)$, which is a sublattice of $\Lambda$, admits three 
possible embeddings up to symmetry as follows:

$$
\begin{gathered}
-e_{2}-e_{3}-e_{4},-e_{1}+e_{2},-e_{2}+e_{3}, e_{1}+e_{2}-e_{4} ; \\
-e_{2}-e_{3}-e_{4},-e_{1}+e_{2},-e_{2}+e_{3},-e_{3}+e_{4}+e_{5} ; \\
\text { or } \quad e_{1}+e_{4}+e_{5},-e_{1}+e_{2},-e_{2}+e_{3},-e_{3}+e_{6}+e_{7} .
\end{gathered}
$$

The first of these does not extend to an embedding of $\Lambda(3,2,2,3,2)$ or $\Lambda(3,2,2,3,3)$ so we discard it. By a simple induction argument, the second of these extends uniquely to an embedding of $\Lambda\left(3^{n-1}, 2,2,3^{n-1}\right)$ as follows:

$$
\begin{aligned}
& -e_{2 n-2}-e_{2 n-1}-e_{2 n}, \ldots,-e_{4}-e_{5}-e_{6},-e_{2}-e_{3}-e_{4},-e_{1}+e_{2}, \\
& -e_{2}+e_{3},-e_{3}+e_{4}+e_{5},-e_{5}+e_{6}+e_{7}, \ldots,-e_{2 n-1}+e_{2 n}+e_{2 n+1} .
\end{aligned}
$$

This can be extended to an embedding of $\Lambda$ in precisely two ways: we may map the additional weight two vertex to $e_{2 n}-e_{2 n+1}$ or to $-e_{2 n+1}+e_{2 n+2}$. The first choice results in an embedding in $\mathbb{Z}^{r}$. The second choice results in an embedding in $\mathbb{Z}^{r+1}$. The orthogonal complement in $\mathbb{Z}^{r+1}$ has rank one and so is generated by a vertex $w$. We may compute $w$ and hence its square directly or use the fact that $\Lambda$ is a primitive sublattice of $\mathbb{Z}^{r+1}$ with determinant $F(2 n+1)^{2}$, which is therefore also the determinant of its rank one orthogonal complement.

Finally another simple induction argument shows that the third embedding in (7) extends uniquely to $\Lambda\left(3^{n-1}, 2,2,3^{n-1}\right)$, and also extends uniquely up to symmetry to give the following embedding of $\Lambda$ :

$$
\begin{gathered}
e_{4 n-7}+e_{4 n-4}-e_{4 n-3}, \ldots, e_{5}+e_{8}-e_{9}, e_{1}+e_{4}-e_{5},-e_{1}+e_{2},-e_{2}+e_{3}, \\
-e_{3}+e_{6}+e_{7},-e_{7}+e_{10}+e_{11}, \ldots,-e_{4 n-5}+e_{4 n-2}+e_{4 n-1},-e_{4 n-1}+e_{4 n} .
\end{gathered}
$$

We see that each of $e_{1}, \ldots, e_{4 n}$ appears in (8), and therefore has nonzero pairing with the image of this embedding.

Proof of Theorem 2. For the duration of this proof, we denote by $B_{n}$ the rational ball $B_{F(2 n+1), F(2 n-1)}$, and by $C_{n}$ the positive-definite plumbed manifold with the same boundary as $B_{n}$, for each $n \in \mathbb{N}$. For $n=1$, the boundary of the rational ball $B_{1}=B_{2,1}$ is $L(4,1)$, and the plumbing $C_{1}$ has weights $[2,2,2]$. For $n>1, C_{n}$ is the plumbing with weights $\left[3^{n-1}, 2,2,3^{n-1}, 2\right]$, as may be seen using [8, Lemma 3.1].

Suppose first that $B_{1} \sqcup B_{n}$ embeds smoothly in $\mathbb{C P}^{2}$. Let $r_{1}=3$ and $r_{2}$ denote the ranks of $\Lambda_{C_{1}}$ and $\Lambda_{C_{n}}$ respectively. By Proposition 3.2, there is a finite-index lattice embedding

$$
\Lambda_{M} \oplus \Lambda_{C_{1}} \oplus \Lambda_{C_{n}} \hookrightarrow \mathbb{Z}^{m}
$$

where $m=r_{1}+r_{2}+1=r_{2}+4$. By Lemma 3.5, the restriction of this to $\Lambda_{C_{1}}$ is either contained in a $\mathbb{Z}^{3}$ or is contained in a $\mathbb{Z}^{4}$, spanned by $e_{1}, \ldots, e_{4}$ say, with orthogonal complement spanned by a vector $w$ of self-pairing 4 . Since the image of the generator of $\Lambda_{M}$ is orthogonal to the image of $\Lambda_{C_{1}}$ and has nonzero pairing with every unit 
vector in $\mathbb{Z}^{m}$ by Proposition 3.2, it must be the second possibility. The image of $\Lambda_{C_{2}}$ lies in the orthogonal complement to that of $\Lambda_{C_{1}}$. If it is contained in the span of $e_{5}, \ldots, e_{m}$ then this is a finite-index embedding in $\mathbb{Z}^{r_{2}}$ which again contradicts the fact that the image of the generator of $\Lambda_{M}$ has nonzero pairing with every unit vector. Thus at least one vertex of $\Lambda_{C_{2}}$ contains a nonzero multiple of $w$. This vertex then has self-pairing greater than that of $w$, contradicting the fact that the vertices of $\Lambda_{C_{2}}$ all have self-pairing 2 or 3 .

We next suppose that $B_{k} \sqcup B_{n}$ embeds smoothly in $\mathbb{C P}^{2}$ with $n \geq k>1$. Let $r_{1}=2 k+1$ and $r_{2}=2 n+1$ denote the ranks of $\Lambda_{C_{k}}$ and $\Lambda_{C_{n}}$ respectively. By Proposition 3.2, there is a finite-index lattice embedding

$$
\Lambda_{M} \oplus \Lambda_{C_{k}} \oplus \Lambda_{C_{n}} \hookrightarrow \mathbb{Z}^{m}
$$

where $m=r_{1}+r_{2}+1=2 k+2 n+3$.

Arguing as in the previous case, we see that the restriction of this embedding to $\Lambda_{C_{k}}$ (respectively $\Lambda_{C_{n}}$ ) cannot have image in either $\mathbb{Z}^{r_{1}}$ or $\mathbb{Z}^{r_{1}+1}$ (respectively $\mathbb{Z}^{r_{2}}$ or $\mathbb{Z}^{r_{2}+1}$ ). By Lemma 3.5, this leaves the possibility that the restriction to $\Lambda_{C_{k}}$ lies in a $\mathbb{Z}^{4 k}$ sublattice, and similarly the restriction to $\Lambda_{C_{n}}$ lies in a $\mathbb{Z}^{4 n}$ sublattice, in both cases with the orthogonal complement in said sublattice containing no unit vectors. In particular we have

$$
4 n \leq 2 k+2 n+3
$$

and hence $n$ is either $k$ or $k+1$.

If $n=k+1$, we have $m=4 n+1$. Up to $\operatorname{Aut}\left(\mathbb{Z}^{m}\right)$, we may suppose that the $\mathbb{Z}^{4 n-4}$ sublattice containing the image of $\Lambda_{C_{k}}$ includes the vectors $-e_{1}+e_{2},-e_{2}+e_{3}$ as the image of the two adjacent weight two vertices. The $\mathbb{Z}^{4 n}$ sublattice of $\mathbb{Z}^{4 n+1}$ containing the image of $\Lambda_{C_{n}}$ has to intersect the $\mathbb{Z}^{3}$ sublattice spanned by $e_{1}, e_{2}, e_{3}$ nontrivially. This means that some vertex of $\Lambda_{C_{n}}$ maps to a vector of the form $v+a\left(e_{1}+e_{2}+e_{3}\right)$, where $v$ is a nonzero vector in the span of $e_{4}, \ldots, e_{m}$ and $a \neq 0$, noting that the image of this vertex is orthogonal to $-e_{1}+e_{2},-e_{2}+e_{3}$ and has pairing -1 with a neighbouring vertex. This contradicts the fact that all vertices in $\Lambda_{C_{n}}$ have weight 2 or 3 .

Finally if $n=k$ then $m=4 n+3$. We keep the notation $\Lambda_{C_{n}}$ and $\Lambda_{C_{k}}$ to distinguish the two copies of $\Lambda_{C_{n}}$. We may suppose that the $\mathbb{Z}^{4 n}$ sublattice containing the image of $\Lambda_{C_{k}}$ is the span of $e_{1}, \ldots, e_{4 n}$, and that it includes the vectors $-e_{1}+e_{2},-e_{2}+e_{3}$ as the image of the two adjacent weight two vertices. Arguing as in the case $n=k+1$, the image of $\Lambda_{C_{n}}$ has to be orthogonal to the span of $e_{1}, e_{2}, e_{3}$, and so is contained in the span of $e_{4}, \ldots, e_{m}$. We may also suppose that the two adjacent weight two vertices in $\Lambda_{C_{n}}$ map to $-e_{4 n+1}+e_{4 n+2},-e_{4 n+2}+e_{4 n+3}$. We consider the image of $\Lambda_{C_{n}}$ and $\Lambda_{C_{k}}$ under the projection to $\mathbb{Z}^{4 n-3}$ spanned by $e_{4}, e_{5}, \ldots, e_{4 n}$. From (8) we see that each of these is isomorphic to the orthogonal direct sum $\Lambda\left(3^{n-2}, 2\right) \oplus \Lambda\left(2,3^{n-2}, 2\right)$, and has rank $2 n-1$. This leads to a contradiction, since it is not possible to orthogonally embed two lattices of rank $2 n-1$ in $\mathbb{Z}^{4 n-3}$. 


\section{REFERENCES}

1. Martin Aigner, Markov's theorem and 100 years of the uniqueness conjecture, Springer, Cham, 2013.

2. Andrew J. Casson and John L. Harer, Some homology lens spaces which bound rational homology balls, Pacific J. Math. 96 (1981), no. 1, 23-36.

3. S. K. Donaldson, The orientation of Yang-Mills moduli spaces and 4-manifold topology, J. Differential Geom. 26 (1987), no. 3, 397-428.

4. Jonathan David Evans and Ivan Smith, Markov numbers and Lagrangian cell complexes in the complex projective plane, Geom. Topol. 22 (2018), no. 2, 1143-1180.

5. Paul Hacking and Yuri Prokhorov, Smoothable del Pezzo surfaces with quotient singularities, Compos. Math. 146 (2010), no. 1, 169-192.

6. János Kollár, Is there a topological Bogomolov-Miyaoka-Yau inequality?, Pure Appl. Math. Q. 4 (2008), no. 2, Special Issue: In honor of Fedor Bogomolov. Part 1, 203-236.

7. Stefan Nemirovski and Kyler Siegel, Rationally convex domains and singular Lagrangian surfaces in $\mathbb{C}^{2}$, Invent. Math. 203 (2016), no. 1, 333-358.

8. Brendan Owens, Equivariant embeddings of rational homology balls, Q. J. Math. 69 (2018), no. 3, 1101-1121.

9. H. Park, D. Shin, and G. Urzúa, Simple embeddings of rational homology balls and antiflips, arXiv:1904.04927, 2019.

School of Mathematics and Statistics

UNIVERSITY OF GLASGOW

Glasgow, G12 8QQ, United Kingdom

Email address: brendan.owens@glasgow.ac.uk 